\title{
HAUSDORFF TRANSFORMATIONS FOR DOUBLE SEQUENCES
}

BY C. R. ADAMS

1. Introduction. The purpose of this note is to extend to double sequences some of the results of Hausdorff's notable papers on methods of summability and moment-sequences. $\dagger$

Let $\lambda=\left\|\lambda_{p q m n}\right\|,(p, q, m, n=0,1,2, \cdots)$, be a four-dimensional matrix of real or complex numbers. Then the system of equations

$$
A_{p q}=\sum_{m, n=0}^{\infty} \lambda_{p q m n} a_{m n},
$$

if the series all converge, transforms a double sequence $\left\{a_{m n}\right\}$ into a new double sequence $\left\{A_{p q}\right\}$. Necessary and sufficient conditions $\ddagger$ that this transformation be convergence-preserving for bounded sequences are the following :

$$
\begin{array}{ll}
\sum_{m, n=0}^{\infty}\left|\lambda_{p q m n}\right| \leqq M, & (p, q=0,1,2, \cdots), \\
\lim _{p, q \rightarrow \infty} \sum_{m, n=0}^{\infty} \lambda_{p q m n}=l, & (m, n=0,1,2, \cdots), \\
\lim _{p, q \rightarrow \infty} \lambda_{p q m n}=l_{m n}, & \\
\lim _{p, q \rightarrow \infty} \sum_{m=0}^{\infty}\left|\lambda_{p q m n}-l_{m n}\right|=0, \quad(n=0,1,2, \cdots),
\end{array}
$$

$\dagger$ Hausdorff, Summationsmethoden und Momentfolgen, I, II, Mathematische Zeitschrift, vol. 9 (1921), pp. 74-109, 280-299.

¥ See Robison, Divergent double sequences and series, Transactions of this Society, vol. 28 (1926), pp. 50-73, especially pp. 71-72. Such transformations are sometimes, if not always, convergence-preserving for certain unbounded sequences; see Adams, Transformations of double sequences, with application to Cesàro summability of double series, this Bulletin, vol. 37 (1931), pp. 741-748; Lösch, Über den Permanenzsatz gewisser Limitierungsverfahren für Doppelfolgen, Mathematische Zeitschrift, vol. 34 (1931), pp. 281-290; Adams, On summability of double series, Transactions of this Society, vol. 34 (1932), pp. 215-230, hereafter referred to as A; and Agnew, On summability of double sequences, American Journal of Mathematics, vol. 54 (1932), pp. 648-656. 


$$
\lim _{p, q \rightarrow \infty} \sum_{n=0}^{\infty}\left|\lambda_{p q m n}-l_{m n}\right|=0, \quad(m=0,1,2, \cdots) .
$$

A matrix $\lambda$ satisfying these conditions we call, with Hausdorff, a C-matrix. Such a matrix defines a transformation (1) which carries a bounded sequence $\left\{a_{m n}\right\}$ convergent to $\alpha$ into a bounded sequence $\left\{A_{p q}\right\}$ convergent to

$$
l \alpha+\sum_{m, n=0}^{\infty} l_{m n}\left(a_{m n}-\alpha\right)
$$

this double series being always absolutely convergent. For a $C$-matrix to define a transformation regular for bounded null sequences it is necessary and sufficient that all the $l_{m n}$ vanish; in such a case the $C$-matrix will be called pure. For a pure $C$ matrix to define a transformation regular for all bounded sequences it is necessary and sufficient that $l=1$; in this event the $C$-matrix will be called normalized.

Now let $\rho$ be a fixed matrix having an inverse and $\mu=\left\|\mu_{m n k l}\right\|$ an arbitrary "diagonal" matrix; that is,

$$
\mu_{m n k l}=0 \text {, }
$$

for $k \neq m$ or $l \neq n$ or both, so that the only elements $\neq 0$ are $\mu_{m n m n}(m, n=0,1,2, \cdots)$. For simplicity we write

$$
\mu_{m n m n}=\mu_{m n} .
$$

Henceforth we restrict ourselves to matrices $\lambda$ of the form $\dagger$

$$
\lambda=\rho^{-1} \cdot \mu \cdot \rho,
$$

which by means of the fixed $\rho$ are transformable into diagonal matrices. It is seen at once that any two such matrices are permutable; moreover, the proof is immediate that if $\lambda^{*}=\rho^{-1} \cdot \mu^{*} \cdot \rho$ is one of the matrices (2) for which the corresponding diagonal matrix $\mu^{*}$ has elements $\mu_{m n}^{*}$ no two of which are equal, then all matrices $\lambda$ permutable with $\lambda^{*}$ are of the form (2).

The system of equations (1) may be written in matrix form as

$$
A=\lambda \cdot a,
$$

where $a=\left\|a_{m n k l}\right\|$ and $A=\left\|A_{p q m n}\right\|$, with

$\dagger$ By a product such as $\lambda \cdot \mu$ we mean the matrix whose element with indices $p, q, k ; l$ is $\sum_{m, n=0}^{\infty} \lambda_{p q m n} \mu_{m n k l}$. 
$a_{m n k l}=\left\{\begin{array}{l}a_{m n} \text { for } k=l=0, \\ 0 \text { otherwise }\end{array} \quad A_{p q m n}=\left\{\begin{array}{l}A_{p q} \text { for } m=n=0, \\ 0 \text { otherwise }\end{array}\right.\right.$

If we set $b=\rho \cdot a, B=\rho \cdot A$, the matrix equation (3) becomes

$$
B=\mu \cdot b, \quad \text { or } \quad B_{m n}=\mu_{m n} b_{m n},
$$

a multiplication. To the matrix $\lambda$ there corresponds a unique diagonal matrix $\mu$, or factor sequence $\left\{\mu_{m n}\right\}$, and conversely. If $\lambda$ is a $C$-matrix, we call $\left\{\mu_{m n}\right\}$ a $C$-sequence; a $C$-sequence will be said to be pure or normalized according as $\lambda$ is pure or normalized.

2. Difference Sequences. From a double sequence $\left\{a_{m n}\right\}$ one may form the quadruple sequence of double differences of various orders,

$$
\Delta_{i j} a_{m n}=\sum_{k, l=0}^{i, j}(-1)^{k+l}\left(\begin{array}{l}
i \\
k
\end{array}\right)\left(\begin{array}{l}
j \\
l
\end{array}\right) a_{m+k, n+l} .
$$

The recursion formulas

$$
\begin{aligned}
\Delta_{i j} a_{m n} & =\Delta_{i j} a_{m+1, n}+\Delta_{i+1, j} a_{m n} \\
& =\Delta_{i j} a_{m, n+1}+\Delta_{i, j+1} a_{m n}
\end{aligned}
$$

may at once be derived. For brevity we call the double sequence of numbers

$$
\Delta_{i j} a_{00}=b_{i j}=\Delta_{00} b_{i j}
$$

the difference sequence of the $a_{m n}$, and from (6) readily follows the relation

$$
\Delta_{i j} a_{m n}=\Delta_{m n} b_{i j}
$$

so that the $a_{m n}=\Delta_{00} a_{m n}$ also constitute the difference sequence of the $b_{i j}$. The matrix associated with (5) is the matrix of the Euler transformation for double sequences,

$$
E=E^{\prime} \odot E^{\prime}
$$

where $E^{\prime}$ denotes the Euler matrix for simple sequence transformations and a notation employed in $A$ is used. $†$ By Theorem

$\dagger$ That is, if each element $a_{m n k l}$ of a four-dimensional matrix $a$ is equal to $a_{m k}^{\prime} \cdot a_{n l}^{\prime}$, where these factors are the elements of two-dimensional matrices $a^{\prime}$ and $a^{\prime \prime}$, we write $a=a^{\prime} \odot a^{\prime \prime}$. 
7 of $A, E$ is its own inverse. We now choose $E$ to be the fixed matrix $\rho$ introduced above, and hereafter consider only such systems of equations (1) as have their difference sequences $b_{m n}, B_{m n}$ in the multiplicative form (4).

We may express the equations (4) in terms of $a_{m n}, A_{p q}$ and obtain without difficulty from $A=E \cdot B$ the relation

$$
A_{p q}=\sum_{i, j=0}^{p, q}\left(\begin{array}{c}
p \\
i
\end{array}\right)\left(\begin{array}{c}
q \\
j
\end{array}\right)\left[\Delta_{p-i, q-j} \mu_{i j}\right] a_{i j}
$$

Setting all the $a_{i j}=1$, we have

$$
\sum_{i, j=0}^{p, q}\left(\begin{array}{c}
p \\
i
\end{array}\right)\left(\begin{array}{c}
q \\
j
\end{array}\right) \Delta_{p-i, q-j} \mu_{i j}=\Delta_{00} \mu_{00}=\mu_{00} .
$$

Then the conditions (A)-(E) become

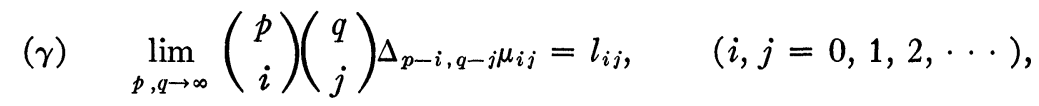

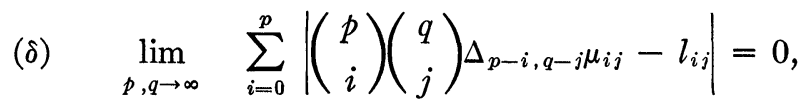

$$
(j=0,1,2, \cdots),
$$

(є) $\quad \lim _{p, q \rightarrow \infty} \sum_{j=0}^{q}\left|\left(\begin{array}{c}p \\ i\end{array}\right)\left(\begin{array}{c}q \\ j\end{array}\right) \Delta_{p-i, q-j} \mu_{i j}-l_{i j}\right|=0$,

$$
(i=0,1,2, \cdots)
$$

the condition $(\beta)$ corresponding to (B) being automatically satisfied because of (7). These conditions $(\alpha)-(\epsilon)$ are thus necessary and sufficient for a $C$-sequence. A $C$-sequence is pure if and only if all the $l_{i j}$ vanish; a pure $C$-sequence is normalized if and only if $\mu_{00}=1$.

The corresponding conditions, necessary and sufficient for a simple sequence $\left\{\mu_{m}\right\}$ to be a $C$-sequence, are 


$$
\begin{gathered}
\sum_{i=0}^{p}\left(\begin{array}{c}
p \\
i
\end{array}\right)\left|\Delta_{p-i} \mu_{i}\right|=M_{p} \leqq M, \quad\left(M_{p} \rightarrow M\right), \\
\lim _{p \rightarrow \infty}\left(\begin{array}{c}
p \\
i
\end{array}\right) \Delta_{p-i} \mu_{i}=l_{i}, \quad(i=0,1,2, \cdots) .
\end{gathered}
$$

Of these the first implies the second with $l_{i}=0$ for $i>0$. It is therefore natural to inquire to what extent condition $(\alpha)$ implies the three remaining conditions $(\gamma)-(\epsilon)$. The complete answer to this question will be given in $\$ 4$, although even in $\$ 3$ it will become apparent that $(\alpha)$ does not imply $(\delta)$ and $(\epsilon)$.

3. C-Sequences of Product Type. An interesting class of matrices $\lambda$ of the form (2) is that for which the diagonal matrix corresponding to $\lambda$ is the product (see the last footnote above) of (simple) diagonal matrices. The importance of this class is sufficiently indicated by the fact that, by Theorem 6 of $A$, this class is identical with the class of matrices $\lambda=\lambda^{\prime} \odot \lambda^{\prime \prime}$, in which $\lambda^{\prime}$ and $\lambda^{\prime \prime}$ are transformable by $E^{\prime}$ into diagonal matrices.

If $\left\{\mu_{m n}\right\}=\left\{\mu_{m}^{\prime} \mu_{n}^{\prime \prime}\right\}$, we clearly have

$$
\Delta_{p-i, q-j} \mu_{i j}=\Delta_{p-i} \mu_{i}^{\prime} \Delta_{q-j} \mu_{j}^{\prime \prime} .
$$

The following theorems may now be established easily.

THEOREM 1. A double sequence $\left\{\mu_{m n}\right\}=\left\{\mu_{m}^{\prime} \mu_{n}^{\prime \prime}\right\}$, where $\left\{\mu_{m}^{\prime}\right\}$ and $\left\{\mu_{n}^{\prime \prime}\right\}$ are (simple) C-sequences, is a (double) C-sequence if and only if the following condition is satisfied:

$$
\left|l_{0}^{\prime}\right|\left(M^{\prime \prime}-\left|l_{0}^{\prime \prime}\right|\right)=\left|l_{0}^{\prime \prime}\right|\left(M^{\prime}-\left|l_{0}^{\prime}\right|\right)=0 \text {. }
$$

Here the $M$ and $l_{0}$ of $(\mathfrak{U})$ and $(\mathfrak{S})$ are primed to agree with the priming of the $\mu$ 's. Any double sequence thus factorable into simple $C$-sequences obviously satisfies $(\alpha)$, but it will satisfy both $(\delta)$ and $(\epsilon)$ if and only if $(8)$ is also fulfilled.

THEOREM 2. A double sequence $\left\{\mu_{m n}\right\}=\left\{\mu_{m}^{\prime} \mu_{n}^{\prime \prime}\right\}$, where $\left\{\mu_{m}^{\prime}\right\}$ and $\left\{\mu_{n}^{\prime \prime}\right\}$ are (simple) C-sequences, is a pure (double) $C$-sequence if and only if one of its factors is a pure $C$-sequence and the other is a pure $C$-sequence or satisfies the condition $M$ $=\left|l_{0}\right|$.

ThEOREM 3. If $\left\{\mu_{m n}\right\}=\left\{\mu_{m}^{\prime} \mu_{n}^{\prime \prime}\right\}$ is a (double) C-sequence that is not pure, both of its factors are $C$-sequences, neither factor is pure, and $l_{0}^{\prime} l_{0}^{\prime \prime}$ equals $l_{00}$. 
Theorem 4. If $\left\{\mu_{m n}\right\}=\left\{\mu_{m}^{\prime} \mu_{n}^{\prime \prime}\right\}$ is a pure (double) C-sequence, not all of whose elements are zero, both of its factors are $C$ sequences and at least one of them is pure.

4. The Relation Between C-Sequences and Moment-Sequences. Let $\chi(u, v)$ be a function which in the square $U(0 \leqq u \leqq 1$, $0 \leqq v \leqq 1)$ is of bounded variation in the sense of Hardy-Krause; then the sequence $\left\{\mu_{m n}\right\}$, where

$$
\mu_{m n}=\int_{0}^{1} \int_{0}^{1} u^{m_{v} n} d_{u} d_{v} \chi(u, v), \quad(m, n=0,1,2, \cdots),
$$

may be termed a (double) moment-sequence. $\dagger$ According to a theorem of Hildebrandt and Schoenberg, $\ddagger$ if $\left\{\mu_{m n}\right\}$ is any double sequence satisfying condition $(\alpha)$ for $q=p$, there exists a function $\chi(u, v)$ which generates this sequence, and conversely; hence we have the following theorem.

TheOREM 5. Every C-sequence is a moment-sequence.

Clearly not every moment-sequence is a $C$-sequence; for otherwise, by Hildebrandt and Schoenberg's theorem, condition $(\alpha)$ for $q=p$ would imply both $(\delta)$ and $(\epsilon)$, and we have already seen that this is not so. We therefore seek to determine under what circumstances a moment-sequence is a $C$-sequence.

It should be observed first that since $\chi(u, v)$ can always be expressed as the difference between two functions $\chi_{1}(u, v)$ and $\chi_{2}(u, v)$, each of which is bounded, is non-decreasing in $x$ alone and in $y$ alone, and satisfies the condition $\chi_{i}\left(u_{1}, v_{1}\right)-\chi_{i}\left(u_{1}, v_{2}\right)$ $-\chi_{i}\left(u_{2}, v_{1}\right)+\chi_{i}\left(u_{2}, v_{2}\right) \geqq 0$ for $u_{2}>u_{1}, v_{2}>v_{1}$; and since the difference (or sum) of two $C$-sequences is a $C$-sequence, no loss of generality results from assuming $\chi(u, v)$ to be of this monotonic character. Then we have, for all $i, j, m, n$,

$\dagger$ Although apparently it is more general to assume only that $\chi(u, v)$ is of bounded variation in the sense of Vitali, this is not actually so; for if $\chi(u, v)$ is of bounded variation in the Vitali sense, there always exists a related function $\chi^{\prime}(u, v)$, where $\chi^{\prime}(u, v)=\chi(u, v)-\chi(u, 0)-\chi(0, v)+\chi(0,0)$, such that the Riemann-Stieltjes integral (9) taken with respect to $\chi^{\prime}(u, v)$ has the same value, and $\chi^{\prime}(u, v)$ is of bounded variation in the sense of Hardy-Krause. Moreover, $\chi^{\prime}(u, v)$ vanishes along $u=0$ and $v=0$; hence it would be no real restriction to assume $\chi(u, 0) \equiv \chi(0, v) \equiv 0$. We do not make this assumption, however, for reasons of symmetry.

$\ddagger$ Hildebrandt and Schoenberg, On linear functional operations and the moment problem for a finite interval in one or several dimensions, Annals of Mathematics, (2), vol. 34 (1933), pp. 317-328, Theorem 1. 


$$
\Delta_{i j} \mu_{m n}=\int_{0}^{1} \int_{0}^{1} u^{m} v^{n}(1-u)^{i}(1-v)^{j} d_{u} d_{v} \chi(u, v) \geqq 0,
$$

so that $\left\{\mu_{m n}\right\}$ is completely monotonic, and by (7), condition $(\alpha)$ is satisfied for all $p, q$. We shall now show that the remaining conditions $(\gamma)-(\epsilon)$ are fulfilled in case $\chi(u, v)$ satisfies the continuity condition

$$
\begin{aligned}
& \chi(u,+0)=\chi(u, 0), \quad \chi(u,+0)=\lim _{v \rightarrow 0} \chi(u, v), \quad 0 \leqq u \leqq 1 ; \\
& \chi(+0, v)=\chi(0, v), \quad \chi(+0, v)=\lim _{u \rightarrow 0} \chi(u, v), \quad 0 \leqq v \leqq 1 .
\end{aligned}
$$

For all values of $u$ and $v$ in $U$ we have

$$
\begin{aligned}
\left(\begin{array}{c}
p \\
i
\end{array}\right) u^{i}(1-u)^{p-i} & \leqq \sum_{i=0}^{p}\left(\begin{array}{c}
p \\
i
\end{array}\right) u^{i}(1-u)^{p-i} \\
& =[u+(1-u)]^{p}=1
\end{aligned}
$$

together with a like relation in $v$. Hence, for $0<\delta<1$, we obtain

$$
\begin{aligned}
\left(\begin{array}{c}
p \\
i
\end{array}\right)\left(\begin{array}{c}
q \\
j
\end{array}\right) \Delta_{p-i, q-j} \mu_{i j} \leqq & \int_{0}^{\delta} \int_{0}^{1} d_{u} d_{v} \chi(u, v) \\
& +\left(\begin{array}{c}
p \\
i
\end{array}\right)(1-\delta)^{p-i} \int_{\delta}^{1} \int_{0}^{1} d_{u} d_{v} \chi(u, v) .
\end{aligned}
$$

Consequently, for each $\delta$, we have

$$
\begin{aligned}
\varlimsup_{p, q \rightarrow \infty}\left(\begin{array}{c}
p \\
i
\end{array}\right)\left(\begin{array}{l}
q \\
j
\end{array}\right) \Delta_{p-i, q-j} \mu_{i j} \\
\qquad \chi(\delta, 1)-\chi(0,1)-\chi(\delta, 0)+\chi(0,0),
\end{aligned}
$$

and so, by virtue of (10), the limit exists and equals zero for each $i$ and each $j$. Thus condition $(\gamma)$ is satisfied with all $l_{i j}=0$. That $(\delta)$ and $(\epsilon)$ are fulfilled then follows in a precisely similar manner, proper account being taken of the relations (11).

When $\chi(u, v)$ does not satisfy condition (10), let

$$
\begin{aligned}
& J_{1}(u)=\chi(u,+0)-\chi(u, 0), u>0 ; \\
& J_{2}(v)=\chi(+0, v)-\chi(0, v), \quad v>0 ; \\
& J_{1}(0)=J_{2}(0)=\lim _{\substack{0 \neq u \rightarrow 0 \\
0 \neq t, 0}} \chi(u, v)-\chi\left(\begin{array}{ll}
0 & 0
\end{array}\right) \text {. }
\end{aligned}
$$


Since $\chi(u, v)$ is bounded and monotonic, all the limits involved exist, and $J_{1}(u)$ and $J_{2}(v)$ are functions of bounded variation. Moreover, condition (10) is satisfied by the function

$$
\chi^{*}(u, v)=\chi(u, v)+J(u, v),
$$

where

$$
J(u, v)= \begin{cases}J_{1}(u) & \text { for } v=0 \\ J_{2}(v) & \text { for } u=0 \\ 0 & \text { for } u, v>0\end{cases}
$$

Therefore it remains only for us to investigate the sequence $\left\{\mu_{m n}\right\}$ generated by $J(u, v)$.

From the definition of the integral in (9), we have at once

$$
\begin{array}{ll}
\mu_{00}=J_{1}(0)-J_{1}(1)-J_{2}(1), & \\
\mu_{m 0}=-\int_{0}^{1} u^{m} d J_{1}(u), & (m=1,2,3, \cdots), \\
\mu_{0 n}=-\int_{0}^{1} v^{n} d J_{2}(v), & (n=1,2,3, \cdots), \\
\mu_{m n}=0, & (m, n=1,2,3, \cdots) .
\end{array}
$$

Thus $\left\{\mu_{m 0}\right\},(m=0,1,2, \cdots)$, and $\left\{\mu_{0 n}\right\},(n=0,1,2, \cdots)$, are both (simple) $C$-sequences. By (13) we have

$$
\begin{aligned}
M_{p q}=\sum_{i=0}^{p} & \left(\begin{array}{c}
p \\
i
\end{array}\right)\left|\Delta_{p-i, 0} \mu_{i 0}\right| \\
& +\sum_{j=0}^{q}\left(\begin{array}{c}
q \\
j
\end{array}\right)\left|\Delta_{0, q-j} \mu_{0 j}\right|-\left|\Delta_{p q} \mu_{00}\right| .
\end{aligned}
$$

Both sums are bounded, and the remaining term on the right is also bounded, since

$$
\Delta_{p q} \mu_{00}=\Delta_{p 0} \mu_{00}+\Delta_{0 q} \mu_{00}-\mu_{00}
$$

is the sum of a term depending upon $p$ alone and a term depending upon $q$ alone, each of which tends to a limit as $p$ and $q$ become infinite. Therefore $(\alpha)$ is satisfied.

Condition $(\gamma)$ is obviously satisfied for $i, j>0$, with $l_{i j}=0$. For $i=0, j>0$, the quantity in question reduces to 


$$
\left(\begin{array}{c}
q \\
j
\end{array}\right) \Delta_{0, q-j} \mu_{0 j}
$$

which tends to zero with $1 / q$; for $i>0, j=0$, the situation is similar. For $i=j=0$, the quantity in question reduces to (14), which, as we have already observed, tends to a limit. Therefore $(\gamma)$ is fulfilled, with $l_{i j}=0$ for $i, j \neq 0,0$ and

$$
l_{00}=\lim _{p \rightarrow \infty} \Delta_{p 0} \mu_{00}+\lim _{q \rightarrow \infty} \Delta_{0 q} \mu_{00}-\mu_{00} .
$$

For $j>0$, the sum in $(\delta)$ reduces to the absolute value of (15), which tends to zero with $1 / q$. For $j=0$, the sum reduces to

$$
\left|\Delta_{p q} \mu_{00}-l_{00}\right|+\sum_{i=0}^{p}\left(\begin{array}{c}
p \\
i
\end{array}\right)\left|\Delta_{p-i, 0} \mu_{i 0}\right|-\left|\Delta_{p 0} \mu_{00}\right|,
$$

of which the first term tends to zero but the remaining part in general does not.

For $i>0$, the sum in $(\epsilon)$ reduces to

$$
\left(\begin{array}{c}
p \\
i
\end{array}\right)\left|\Delta_{p-i, 0} \mu_{i 0}\right|
$$

which tends to zero with $1 / p$. For $i=0$, the sum reduces to

$$
\left|\Delta_{p q} \mu_{00}-l_{00}\right|+\sum_{j=0}^{q}\left(\begin{array}{l}
q \\
j
\end{array}\right)\left|\Delta_{0, q-j} \mu_{0 j}\right|-\left|\Delta_{0 q} \mu_{00}\right|,
$$

of which the first term tends to zero but again the remaining part in general does not. The results of this discussion may be summed up as follows.

THEOREM 6. Let $J_{1}(u)$ and $J_{2}(v)$ represent the jump of $\chi(u, v)$ at the sides $v=0$ and $u=0$, respectively, of the square $U$ (for orthogonal approach except at $(0,0)$, where the approach is made in an arbitrary manner from the interior of $U) . J_{1}(u)$ and $J_{2}(v)$ are functions of bounded variation and so generate (simple) moment-sequences which we may denote by $\left\{j_{m}^{(1)}\right\}$ and $\left\{j_{m}^{(2)}\right\}$, respectively. The sequences $j_{0}{ }^{(1)}-J_{2}(1), j_{1}{ }^{(1)}, j_{2}{ }^{(1)}, \cdots$ and $j_{0}{ }^{(2)}-J_{1}(1)=j_{0}{ }^{(1)}-J_{2}(1), j_{1}{ }^{(2)}, j_{2}{ }^{(2)}, \ldots$, are also moment-sequences and therefore $C$-sequences; let the $M$ of (i) and the $l_{0}$ of (C) associated with these sequences be denoted respectively by $M^{(1)}, l_{0}{ }^{(1)}$ and $M^{(2)}, l_{0}^{(2)}$. Then a (double) moment-sequence is a (double) $C$-sequence if and only if we have 


$$
M^{(1)}-\left|l_{0}^{(1)}\right|=M^{(2)}-\left|l_{0}{ }^{(2)}\right|=0 .
$$

$A$ (double) moment-sequence is a pure (double) $C$-sequence if and only if in addition to (16) we have

$$
l_{0}^{(1)}+l_{0}^{(2)}-j_{0}^{(1)}+J_{2}(1)=0 .
$$

In particular we note that if $J_{1}(u) \equiv J_{2}(v) \equiv 0, \chi(u, v)$ generates a pure $C$-sequence.

Theorem 7. Condition ( $\alpha$ ) for $q=p$ implies the entire set of conditions $(\alpha)-(\epsilon)$, including $l_{i j}=0$ for $i, j \neq 0,0$, with the exception of $(\delta)$ for $j=0$ and $(\epsilon)$ for $i=0$.

Although in this section it has been tacitly assumed that the sequences considered are real, the extension of the results to complex sequences is immediate.

BROWN UNIVERSITY

\section{ERRATA}

This Bulletin, vol. 38, No. 12 (Dec., 1932):

Page 841, first formula: inside of the large parentheses in the denominator, the numerator of the small fraction should be $n$ instead of 1 .

Page 847 , equation (11): the quantity $\mathrm{c} / 2$ should be added to the left-handed side.

Page 847, last line: the second and third integrals should be preceded by the negative sign.

This Bulletin, vol. 39, No. 1 (Jan., 1933):

Page 18, line 8: read Kline in place of Kine. 